\title{
Morphological Changes during Uniaxial Drawing and Annealing of Poly(ethylene terephthalate) Films
}

\author{
Leonid I. BezruK*, Tohru Kawai, and Yu. S. Lipatov* \\ Tokyo Institute of Technology, Ookayama, \\ Meguro-ku, Tokyo, Japan.
}

(Received January 14, 1974)

\begin{abstract}
Morphological changes occurring during uniaxial drawing and subsequent annealing of amorphous poly(ethylene terephthalate) films have been studied. In the course of the drawing the polymer molecules or their aggregates were separated in the direction normal to the drawing direction. The original supermolecular structures of crystallite dimensions remained in the state of hexagonal packing. After annealing, the optical contrast of the multilayers in the film plane became more sharp, which may be attributed to a dominating crystallization into the form of lamellae. At the same time a separation of the polymer molecules composing the lamellae was observed in the planes perpendicular to the film plane. The absolute magnitudes of the long spacing determined by microscopy and by X-ray scattering showed a reasonable agreement.

KEY WORDS Morphological Changes / Uniaxial Drawing / Annealing / Poly(ethylene terephthalate) / Small-Angle X-ray Scattering /
\end{abstract}

\section{INTRODUCTION}

It is well-known that the current interest in morphological investigations of polymers is caused mainly by the dependence of some important polymer properties not only on their chemical nature but also on their supermolecular and even more gross (on the dimensional scale) structure. These properties definitely include characteristics of strength and deformation, gas and vapor permeability, swelling, etc. It is possible that dielectric as well as some other properties also depend on polymer morphology.

In general, morphological studies involve two main physical methods, namely, various diffractions of electromagnetic emission (i.e., light, $\mathrm{X}$-rays, electrons, etc.) and microscopy (light, electron). The former method is faster and cheaper, whereas the latter seems to be more attractive due to the "self-consistence" of the picture and to the possibility of the choice of simpler analogies for the interpretation of morphological changes in definite localities of the picture studied. It should be added that

\footnotetext{
* Present address: Institute of Macromolecular Chemistry, Ukrainian Academy of Sciences, Kiev, Kharkovskoe Shausse, 48, U.S.S.R.
}

the diffraction methods are intrinsically more "integral" and consequently, their interpretation is generally not straightforward and is sometimes ambiguous. The very simple considerations known from basic courses in physical optics suggest that it is necessary to compare the diffraction data with the microscopy data, and it is only in the case of their compatibility that we may speak of the correctness of both the microscopy data and diffraction data interpretations. The point of importance is that the process of the formation of the observed optical image in the microscope proceeds through the stages of the formation of a diffraction picture from the object, and it can be recalled that the method of reproduction of such a diffraction picture from the light- or electron-microscopic image has been known for about 150 years. This method is known as a "parallel-rays diffraction", (or Fraunhofer diffraction). On the other hand, it is quite possible to obtain simultaneously the visible image and the low-angle light- or electron-diffraction from the same part of a sample in the course of studies of a certain morphological situation. ${ }^{1,2}$

It may be safely concluded that the early age of fascination with microscopy data, when each 
observed picture was taken as an absolutely ultimate result of the study and used for making revolutionary conclusions, is gone. It has been noticed for a long time that, depending on the method of preparation, one can obtain from the same object very different and sometimes quite opposite microscopy data.

In our opinion, we are presently faced with some very acute and urgent problems, such as the morphological structure of amorphous and crystallizable polymers, as well as more local problems of preparative methods and structure investigations on polymeric materials (especially commercially produced) by means of electron microscopy. We may cite as such local problems the choice of the experimental method as well as the estimation of the experimental resolution of measurement (besides the problems of the correlation and correctness of the data mentioned above).

\section{CHOICE AND REAPPRAISAL OF EXPERIMENTAL METHOD}

Preparation of samples for an electron microscopic study was carried out by treatment of the polymer surface with plasma generated in a linear electrode-less high-frequency discharge ${ }^{3}$ with subsequent replication of a fresh surface. We have chosen this method because the morphological picture of an initial surface of any polymeric material is drastically different from that of inner layers. If we want to obtain microscopy pictures for the purpose of making a correlation with the results of transmission diffraction, we have to remove the "false" surface layer. The striking morphological differences between the polymer surface and the inner layer may be primarily the result of the action of surface tension forces produced during solidification from the polymer solution or the melt. Or they can be the reflection of previous technological processing, when this surface is a "replica" of the surface finish of dies, molds, rollers, etc. Finally, they may be also the result of aging and/or impurity entrapment during storage and so on.

The use of many classical methods of polymer preparation for electron microscopic studies, such as microtomy, cleavage, mechanical disintegra- tion, etc., is inevitably followed by some pronounced changes in the previous morphology. These changes are brought about both by very strong mechanical action on the innermost structure of a sample and by the effect of complementary media which are used to increase the efficiency of the methods used. This latter effect can be arbitrarily divided into a mechanochemical one (swelling, effects of surface-active agents, plasticization, etc.) and a temperaturemechanical one (volume and phase changes during quenching for the cleavage or during high-temperature curing of various amorphous substances used in microscopy and microtomy).

Besides, if such analogies prove to be appropriate, the polymer sample preparation for microscopic work will be similar in some respects to archaeological excavations, since it is only in the case of a very slow and easily controlled removal of a certain layer that high probability of the slightest possible distortion of the initial structure of the sample exists. We believe that the treatment in the plasma of an electrode-less high-frequency discharge has such an attractive property. That is, the energy of plasma particles is so low that the process of chain rupture is located in very thin surface layers. For example, electrons with an energy of about $100-\mathrm{eV}$ leave tracks of only $14 \AA$ (for materials made of light atoms like $\mathrm{H}, \mathrm{C}$, and O). On the other hand, an energy of only about $2.6 \mathrm{eV}$ is sufficient for dissociation of aliphatic $\mathrm{C}-\mathrm{C}$ bonds. According to the existing concepts of the physics of a gas-discharge plasma, moreover, the mean energy of an ion in the plasma is two orders of magnitude lower than that of an electron in the plasma. Therefore, if polymer treatment in plasma is carried out with ions of sufficient energy, the electron tracks might turn out to be very large, and the interaction of the electron component of the plasma with the polymer will cause structural changes in the inner layers of a sample, which is, of course, unsatisfactory.

The next very important parameter of a gas discharge is the plasma particles' concentration. However, this is the easiest parameter to control, and the rate of surface disintergration may be arbitrarily changed by variation of the high- 
frequency generator output or of the gas pressure in the discharge vessel.

The ultimate result of the action of the gasdischarge plasma on the polymer sample surface (which is in a solid state) is the transition of the minute surface layers from the previous aggregated state into a gaseous one, which is a chain rupture. If a dynamic vacuum system is employed, i.e., if the gaseous products of the polymer degradation are continuously evacuated, then we obtain continuous renewal of the sample surface, which is thinning. As far as the structure of any object may be thought of, in the first approximation, as a distribution and/or interconnection of microregions of different natures, the denser regions will first become revealed as a surface relief, then these will in turn degrade and uncover the structure of the more deeply buried layers. In physics such a phenomenon is called "etching". However, another case of very fast sublimation of surface layers is also possible, where the surface relief will not be very clear-cut, but the thinning rate will increase. This can be obtained by increasing the power of the plasma particles' beam, while the required minimum mean particle energy remains unaltered. Usually this latter method is employed in the early stage of treatment for surface "cleaning" and afterwards the "etching", proper is carried out. ${ }^{4}$

Replication was made with self-shadowed carbon replicas. The usual method of shadowing with heavy metals or their salts was avoided because we wanted to obtain simultaneously low-angle electron diffraction and electron micrograph data from the same region of the sample (to search for a correlation). When metals were used, one may eventually deal with the overlap of metal diffraction which will make the interpretation difficult. Since the carbon atom diameter is very small $(1.54 \AA)$, moreover, there are reasons to expect an experimental "translimit" resolution, i.e., beyond the natural limit of the equipment, due to the replication of etched polymer surfaces. Indeed, it is reasonable to assume that in the case of plasma etching of a single crystallite for example the breakdown of molecular planes will not proceed simultaneously, but the crystallite surface itself will be revealed as steps of these planes. The empty space in such steps will be filled (i.e., decorated) with dispersed carbon atoms. This will create the precondition for obtaining diffraction contrast, in addition to that of amplitude, from the regions of widely developed steps.

The replicas were stripped from the sample surface and treated with a 30-\% water solution of gelatin backed with carbon. However, in this case the tearing out of an ultrathin surface layer of the polymer was observed. For electron microscopic studies we used replicas prepared as described above, as well as some obtained by slow (for the order of several minutes) thermal degradation of the polymer in vacuo somewhat above its ordinary degradation point. Since carbon was the only substance used in the preparation of replicas, the danger of distortion of the surface relief was avoided. The mean thickness of the replicas was in the range from 150 to $300 \AA$, which is sufficient for use of direct instrumental magnifications of some hundreds of thousands with adequately high image contrast. The wide-angle electron diffraction technique was used to check whether the replicas were made of "grains", and no measurable crystallization was observed. We estimate the mean size of the grains to be less than $5 \AA$.

A vacuum system deposition unit, JEE-SS of Japan Electron Optics Lab., was used for the plasma treatment (see the corresponding blockdiagram in ref 3) and the Geissler discharger of this unit was used as the high-frequency generator. Air or oxygen was used as the working media in the discharge camera. "Fast"* sublimation was carried out at 0.075 torr and the etching process at 0.03 to 0.01 torr. The rate of the sublimation depends on chemical and physical sample pretreatments. For example, at an identical electron concentration of plasma the sublimation rates for amorphous poly(ethylene terephthalate) (PET) and for crystalline PET (of about $30-\%$ crystallinity) in oxygen are 3.45 and $3.23 \mathrm{mg} / \mathrm{cm}^{2} \mathrm{sec}$, respectively.

* Faster sublimation rates are possible with more powerful high-frequency generators. Methods for estimation of plasma parameters, range of thinning, etc., are given in ref 4 . 
The X-ray diffraction study was made by standard photographic methods using a RIGAKUDENKI RU-3 V unit. The electron microscopy and electron diffraction studies were made with JEM-100B and JEM-7 units.

In this study Tetoron (PET made by TEIJINcompany) films were used. PET samples were drawn at room temperature at a rate of 0.5 $\mathrm{mm} / \mathrm{min}$. In such conditions the draw ratio amounted to 10 to 11 for fresh (raw) films, but afterwards the ultimate elongation dropped to 7 to 8. PET was crystallized in a silicone-oil bath at $190^{\circ} \mathrm{C}$ for $6 \mathrm{hr}$ with subsequent quenching in ice water and washing out of the silicon oil in $n$-hexane. Oriented PET was crystallized in the free (unstrained) state as well as at a fixed length.

\section{MORPHOLOGICAL RESULTS AND DISCUSSION}

$\mathrm{X}$-ray diffraction data show that the orientation of molecules in "amorphous" PET proceeds more easily than that in noncrystallizable polymers (Figure 1). Indeed, after drawing by $50 \%$ two equatorial spots are already observed on wide-angle X-ray pictures, while further drawing (up to $1000 \%$ ) did not result in any additional increase in the scattering intensity or change in the positions of these reflexes. Such behaviour can be attributed only to the existence of supermolecular crystalline-like entities ("bad" or "imperfect" crystallites) in the bulk polymer, which rotate in the orientation field like ele- mentary magnets in a magnetic field. We speak about "crystalline-like type" because in this case an approximately parallel orientation of the macromolecules in the interior of these entities appears to be natural. On the other hand, for noncrystallizable linear polymers such as atactic polystyrene, poly(methyl methacrylate), etc., the orientation of the macromolecules is usually proportional to the draw ratio.

Small-angle X-ray diagrams (Figure 2) show that the mutual arrangement (or maybe the shape?) of the supermolecular structures of this polymer both in the oriented and unoriented "amorphous" state differs only a little; in all cases no discrete scattering was observed. The most plausible interpretation of these data from a structural point of view would be hexagonal packing of the elementary supermolecular entities. In other words, in this case we cannot expect the appearance of microfibrillar and lamellar entities or, at any rate, the concentration of the latter in bulk would be small. Indeed, electron microscopic pictures show the morphology of an "amorphous" state both for unoriented and completely oriented PET (Figure 3). Here, individual supermolecular entities (dimensions of about $100 \AA$ ) and/or their aggregates are generally observed, as was found by Yeh, et $a l^{5}$ In the majority of cases the polymer structure may be represented as hexagonal packing of the individual supermolecular entities (that is, in a cross-sectional view each entity is surrounded by six similar ones). How-

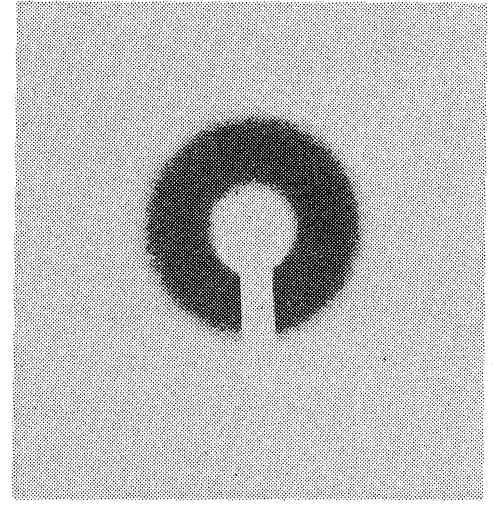

(a)

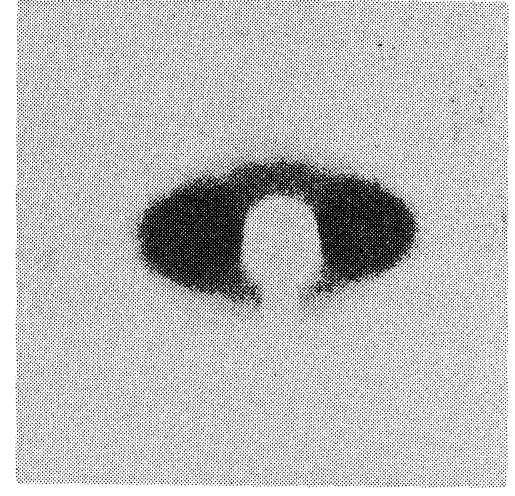

(b)

Figure 1. The changes in wide-angle X-ray diffraction data for amorphous poly(ethylene terephalate): (a), unoriented state; (b), drawn to 3 times. 


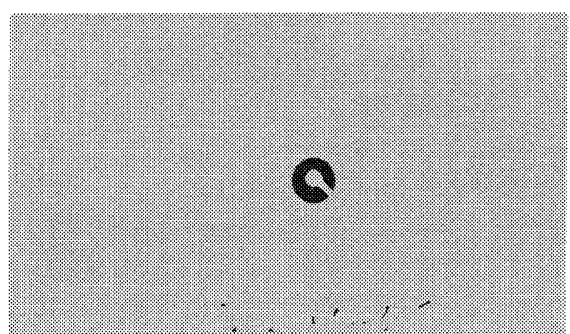

(a)

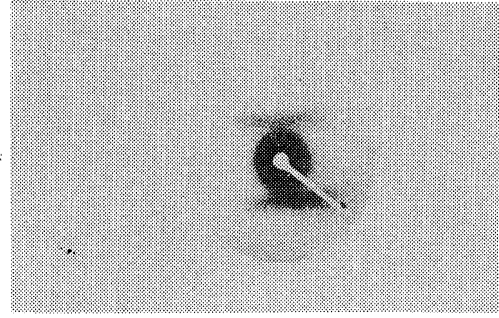

(c)

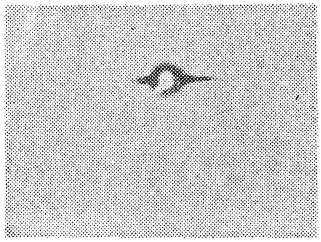

(e)

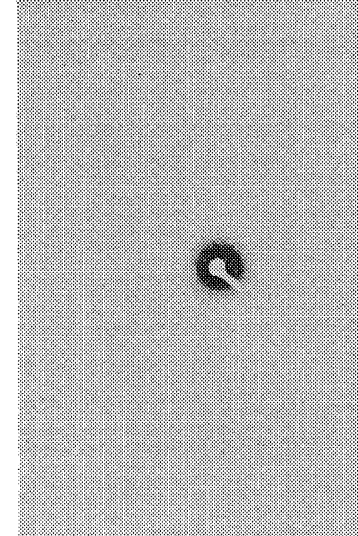

(b)

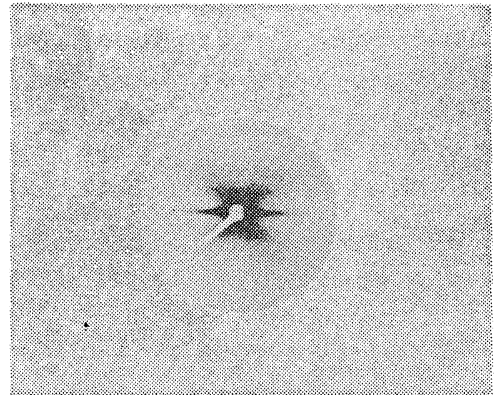

(d)

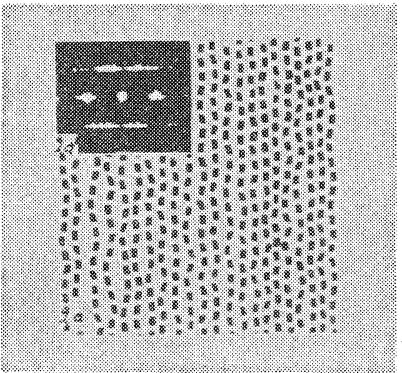

(f)

Figure 2. Low-angle X-ray scattering data for poly(ethylene terephalate) films: (a), amorphous film drawn to 11 times; (b), unoriented crystallized (annealed); (c), scattering in the r-plane (see Figure 4) after crystallizing (annealing) the sample drawn to 11 times; (d), scattering in the t-plane (see Figure 4) of the same sample as (c); (e), scattering in the d-plane (see Figure 4) of the same sample as (c); (f), optical model of the microfibriller structure.

ever, we prefer to mark the "amorphism" of such a PET state with inverted commas, since during melt-processing of PET and particularly in the course of its orientation, the conditions for both a mutual approach of the segments of neighboring chains to their van der Waals distances and the formation of planes of crystalline lattice in imperfect crystallites, as well as similar "sticking" of neighboring crystallities and the formation of primary supercrystalline structures (lamellae), can be realized. Morphologically, the latter are seen in the oriented PET 
samples in the form of small chains (of the order of several microns). These chains are arranged approximately orthogonal to the draw direction, that is, the orientation of the polymer segments is perpendicular to the lamellar surface. This latter assignment could be proved by comparison of the wide-angle X-ray diffraction data with the shadow direction in replicas. As a rule, either the parallel or the normal direction of shadowing with respect to the texture was chosen in order to eliminate arbitrariness in the data interpretation. Probably, these data correspond to crystallization during the stretching

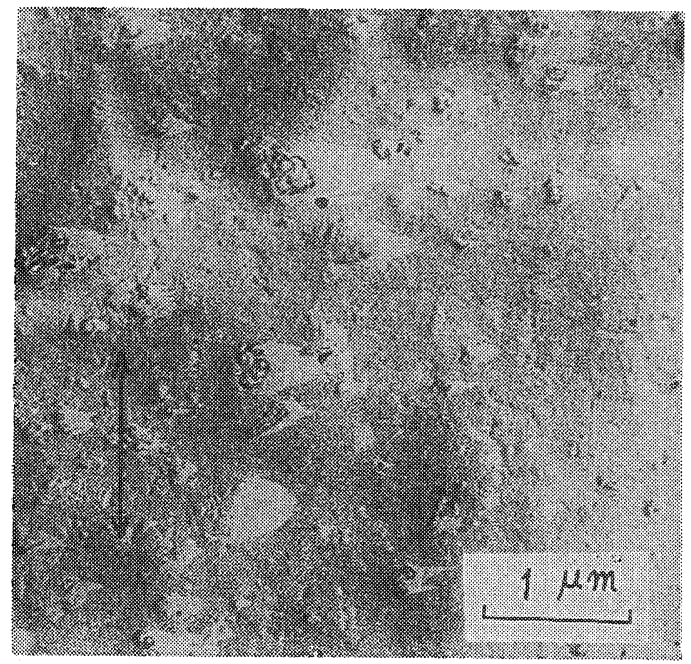

(a)

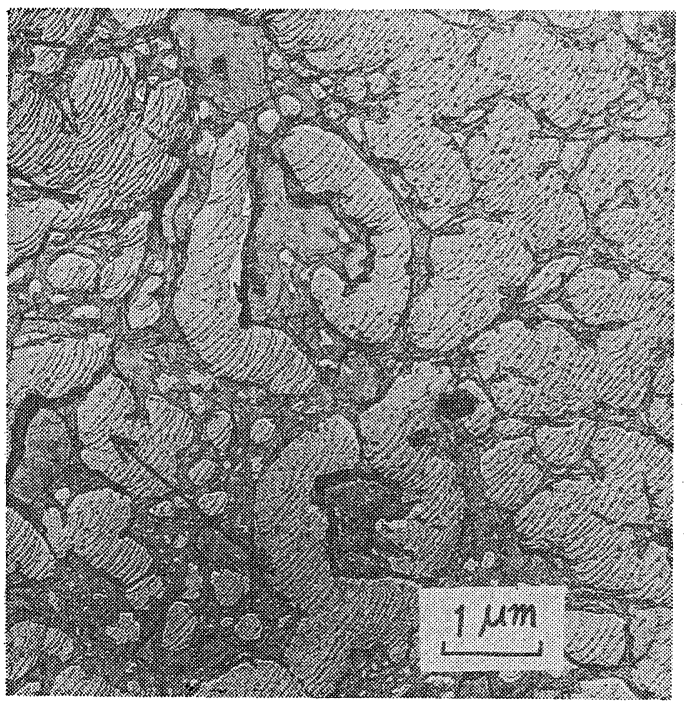

(c) as a mechanically forced process (it is pertinent to recall that the stretching was carried out at the room temperature, which is below the glass transition temperature).

After annealing, changes in both the phase state and the morphology of these samples took place. X-ray (wide-angle) diffraction data have shown a substantial (about 30\%) crystallinity of the unoriented PET and a change in the diffraction spot positions occurred upon annealing in all samples, as compared with the amorphous state. We do not wish to get involved in the discussion of crystallographic details of these

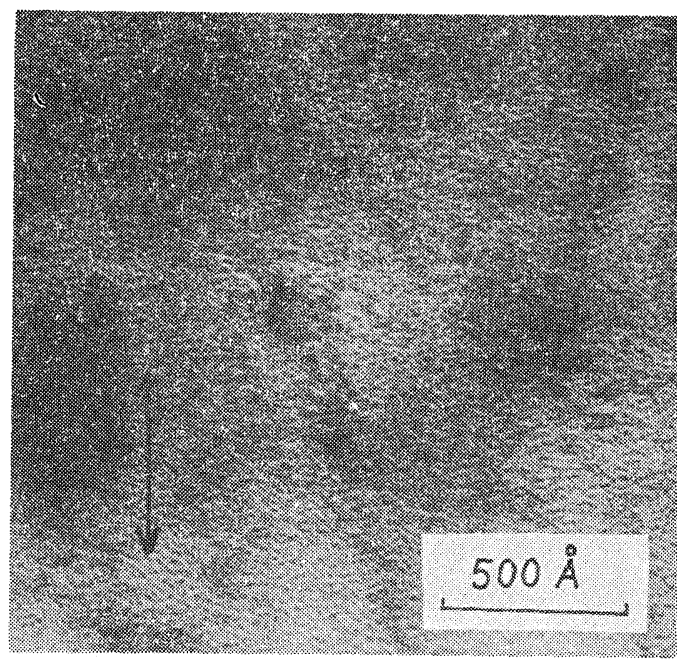

(b)

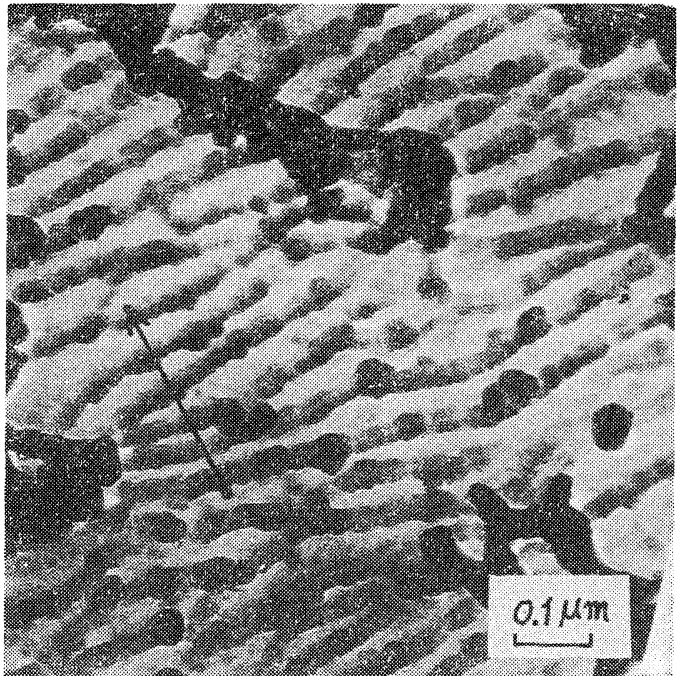

(d) 


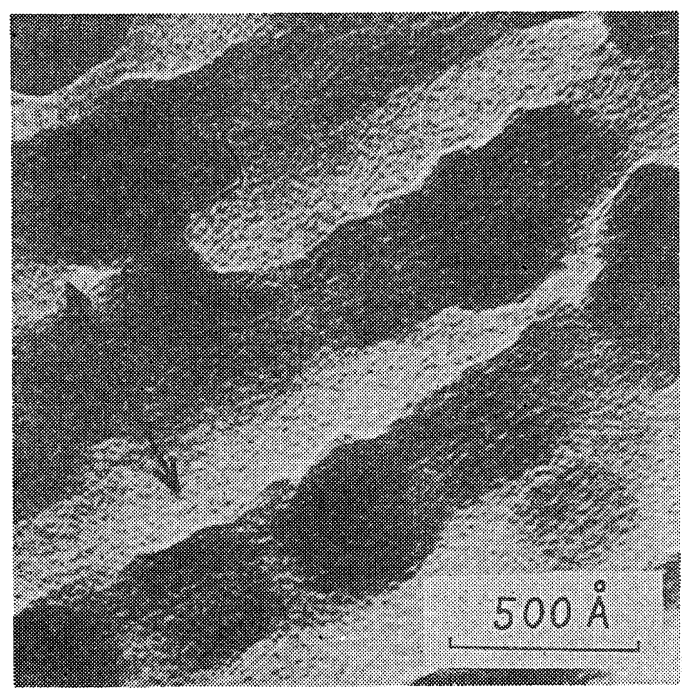

(e)

Figure 3. Morphological changes of poly(ethylene terephthalate) films in the course of crystallization (annealing) by means of electron microscopy: (a) and (b), the difference in morphological view at low and high magnifications for "amorphous" poly(ethylene terephthalate) films drawn to 11 times; (c)-(e), similar differences for the same samples after crystallization (annealing). In Figure 3c it is possible to see the bulk surfaces of the sample-original and etched. In Figure $3 \mathrm{e}$ the fine structure of the multilayers is shown. Arrows indicate the stretching direction.

changes, ${ }^{6}$ since our primary concern now is in the qualitative understanding of the process and the possibility of obtaining a correlation with the microscopic data. In this respect one can note the conservation of rather high molecular orientation even after annealing PET in a free state. Other important features concern the crystallization phenomenon itself. Therefore, one might expect qualitatively some changes in the morphological pictures, i.e., an enhancement of the "cohesion" of the molecular segments in the interior of the crystallites, as well as a transformation of the intercrystallite aggregation into more definite supercrystalline structures. Low-angle X-ray diffraction pictures have proved these expectations to be true (Figure 2). In the case of unoriented and crystallized PET the distinct ring corresponding to the scattering from the dense polymer regions with mean dimension of about $100 \AA$, appeared, ${ }^{7}$ whereas in the oriented PET the meridional spots appeared. The position and nature of these spots were different for different planes of the object (Figure 4d). In the plane most suitable for the microscopy (the plane of the film surface which is arbitrarily designated as the r-plane) we obtained a "fourpoint" scattering picture, where the meridional spots corresponded to the long spacing, that is, a periodicity of the densities of $60 \AA$; no equatorial scattering was observed. Similar pictures were obtained for the samples annealed in both the free and the fixed states. In the plane corresponding to the sample thickness (t-plane) the scattering pattern was different: apart from the former meridional spots, an X-like spot appeared, which corresponded to a different periodicity of about $90 \AA$ along the stretching direction; very strong equatorial scattering also appeared. For the "three-dimensional" analysis of the morphological situation we also obtained the scattering pattern from the plane normal to both of the two planes mentioned above (this is designated as the d-plane). Here we have only equatorial scattering from the series of planes piled in the direction normal to the r-plane.

It follows already from the appearance of meridional spots that we should observe under the microscope a similar periodical series of densities along the stretching direction. On the other hand, different scattering patterns from the 
same object have their origin in the differences in the morphologies in those planes of the sample which were chosen for the scattering. The fact of the appearance of the X-shaped spot in the t-plane in addition to the meridional reflexes in the r-plane would be the reflection of the lamellar nature of new morphological structures in the orienied crystalline PET. Indeed, if we suppose the existence of a microfibrillar structure (as is sometimes assumed for the description of the morphology of oriented crystalline polymers), we should have observed absolutely identical scattering patterns from both of these cross-sections (the r- and t-planes). Besides, if one analyzes the optical model of the microfibrillar structure, then in addition to the arc meridional reflexes we should have point equatorial ones (Figure 2f) from the fibril "diameters". As already mentioned above, equatorial scattering appeared only in the $\mathbf{t}$ - and $\mathbf{d}$ planes, and were more arc-like than spot-like.

Thus, in the schematic models (Figure 4) of the morphological picture in the case of crystallized highly oriented PET, we must take into account the existence, on the one hand, of high molecular orientation, and on the other hand, the absence of equatorial point reflexes in the $\mathbf{r}$ and t-planes, as well as the splitting of the meridional arc reflexes. The latter fact reflects the oblique (to the texture axis) position of the dense regions, of a linear form, in the supercrystallite organization in both the planes, the slope being more pronounced in the $t$-plane. If we also recall the appearance of the system of equatorial are reflexes (we note that the interpretation of the latter scattering is quite obscure, but we cannot neglect it in the present discussion), then as one of several possible explanations we may propose a layer separation in the t-plane: the layers are parallel to the r-plane. Apparently, this separation appears as a necessary consequence of the compensation of the oblique arrangement of the supercrystallite structures in the sample: i.e., in the t-plane the macrolattice has the form of a zig-zag. In our opinion, the appearance of such a zig-zag has nothing to do with the rotation of the supercrystalline structures (lamellae), but rather may proceed via mutual slipping of the crystallites in the lamellae or of the individual planes within the crystallites. If a lamellar rotation had taken place, on the other had, then substantial disordering of macromolecules would be unavoidable; this, however, was not observed.

The microscopic data are in rather good qualitative correspondence with those from lowangle X-ray diffraction (Figure 3). Indeed, the dense layers (lamellae) are arranged normally to the texture. Therefore, we may except substantial meridional scattering on the X-ray pictures. Due to the bias of these layers with respect to the texture axis oblique regions appear, expecially at the junctions between neighboring layers; this leads to the splitting of the corresponding spots. The transverse layer dimensions and the eletronic density in the crystallite boundaries in the interior are comparatively large, which may lead to the virtual absence of equatorial spots. Moreover, the broadening of meridional spots can also be simply explained by the longitudinal (texturewise) lamellar twist.

The low-angle electron diffraction data as well as the analysis of the Fraunhoffer diffractions of some optical models also confirm such an interpretation of the microscopic pictures. However, we must not leave unmentioned the quantitative discrepancy between the results of the electron and X-ray diffractions: the long spacing determined from the former is 4 to 5 times larger than that from the latter. At present the experimental difficulties did not allow us to obtain the electron diffraction with the absolute (both quantitative and qualitative) coincidence of this value, since even when choosing the electron scattering angle corresponding to the quantitative coincidence, some false scattering appeared due to the coarseness of the replica relief.

From the above microscopic data one more practical conclusion can be deduced. Namely, the experimental resolution of the replicas turns out to be better than is usually assumed. This is especially true in the case of widely developed morphological structures, where the effect of diffraction contrasting can be applied. Of course, there is a danger of incorrect estimation of the data from the experimental resolution 


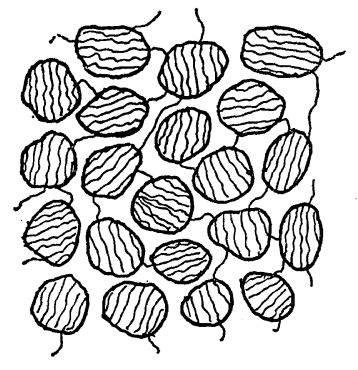

(a)

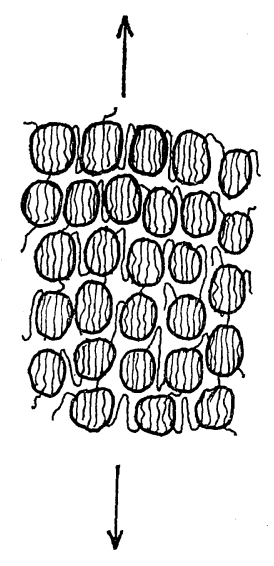

(b)

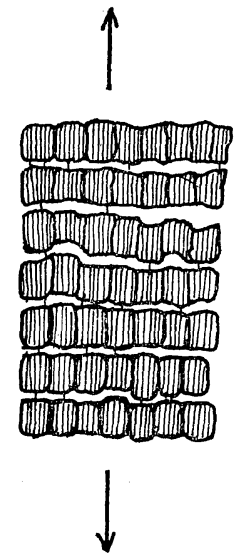

(c)

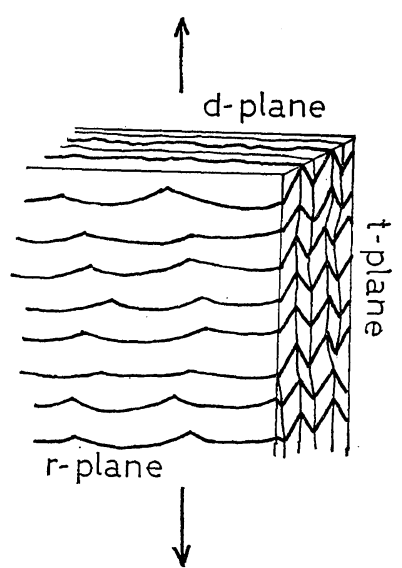

(d)

Figure 4. Schematic representation of changes in the morphological situation in the course of uniaxial stretching and annealing of "amorphous" poly(ethylene terephthalate) films: (a), amorphous unoriented state; (b), amorphous oriented state; (c), annealed oriented state; (d), "three-dimensional" schema of the oriented crystallized state (c).

(this is connected mainly with the "grains" in the replicas and the photographic materials, or with inadequate focusing), but on the other hand, such discrepancies can always be avoided due to simultaneous diffraction control. Moreover, use of only small instrumental magnifications does not allow one to estimate the morphological difference, for example, in the case of crystallized and amorphous oriented PET, due to the fact that newly formed multilayers resulting from the orientation differ only in their finer structure, that is in the mutual arrangement of the crystallites.

\section{CONCLUSION}

Thus, parallel diffraction and microscopic observations permitted us to give a unified interpretation of the morphological changes during the uniaxial orientation of "amorphous" PET and its subsequent crystallization by annealing (see Figure 4). In the course of orientation the polymer molecules separate normally to the texture. Prior to the crystallization by annealing the supermolecular structures of crystallite dimensions have, in general, hexagonal packing. After annealing a sharpening of the optical contrast of the multilayers in the r-plane was observed, which may be attributed to the dominating crystallite-crystallization into lamellae along the planes $\{h 00\}$ or $\{0 k 0\}$. At the same time the separation of the polymer molecules in the $\mathbf{d}$ - and t-planes is noted (at present only by diffraction). The absolute values of the long spacing determined from the microscopy and $\mathrm{X}$-ray scattering show a reasonable agreement.

\section{REFERENCES}

1. A. Ono, K. Ibe, and H. Yotsumoto, J. Electron Microscopy, 19, 149 (1970).

2. Yu. S. Lipatov, L. I. Bezruk, M. P. Nosov, A. P. Ochkivskii, and T.S. Melnik, The reports of Ukrainian Academy of Sci., No. 9B, 829 (1972).

3. L. I. Bezruk, G. A. Gorokhovskii, and Yu. S. Lipatov, Vysokomol. Soedin., Ser. A, 10, 1434 (1968).

4. L. I. Bezruk and E. V. Lebedev, ibid., Ser. A, 15, 1674 (1973).

5. G.S. Y. Yeh and P. H. Geil, J. Macromol. Sci.Phys., B1, 235 (1967).

6. T. Matsumoto, K. Ehara, N. Ikegami, T. Kawai, and H. Maeda, Sen-i Gakkai Shi (J. Soc. Fiber Sci. Technol.), 25, 5 (1969).

7. T. Matsumoto, N. Ikegami, K. Ehara, T. Kawai, and H. Maeda, Kogyo Kagaku Zasshi (J. Chem. Soc. Japan, Ind. Chem. Sect.), 73, 2441 (1970). 